\title{
Achieving Scalability in Project Based Learning through a Low-Code platform
}

\author{
João Paulo Fernandes \\ CISUC, Universidade de Coimbra \\ Coimbra, Portugal \\ jpf@dei.uc.pt
}

\author{
Ricardo Araújo \\ OutSystems \\ Proença-a-Nova, Portugal \\ ricardo.araujo@outsystems.com
}

\author{
Mário Zenha-Rela \\ CISUC, Universidade de Coimbra \\ Coimbra, Portugal \\ mzrela@dei.uc.pt
}

\begin{abstract}
Defining an adequate project for a Software Engineering course is a challenging endeavour. Such a project must simulate as faithfully as possible a real industrial project, while accounting, e.g., for: i) the natural lack of experience of the students, ii) their constraints to full-time dedication, and iii) reasonable effort required from the instructors. Additionally, while having a real client from industry may contribute to a more realistic experience, the project itself must be challenging enough to motivate the client while still not unduly burden the students.

We report on our experience and share our insights from adopting a state-of-the-art low-code software development platform as the core technology for project-based learning -with a real clientin a one-semester software engineering course. We had to handle i) a large class (200+ students), while providing ii) individual assessment iii) for students from very different backgrounds (majoring in three different topics). While we believe i) and ii) are recurrent, iii) poses a significant challenge in the establishment of a fair pedagogic context.

We assess the merit of the experience taking as proxy: i) the students' individual and group performance, assessed both by the instructors and the client, and ii) the results of the course's standard institutional pedagogical survey. We have found evidence that the designed project created an even playing field for students from different backgrounds, while being manageable for the instructors and rewarding for the client.
\end{abstract}

\section{CCS CONCEPTS}

- Software and its engineering $\rightarrow$ Software development techniques; Development frameworks and environments.

\section{KEYWORDS}

Low-Code Platforms, Project-based Learning, Scalability, Software Engineering Education.

ACM Reference Format:

João Paulo Fernandes, Ricardo Araújo, and Mário Zenha-Rela. 2020. Achieving Scalability in Project Based Learning through a Low-Code platform.

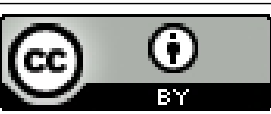

This work is licensed under a Creative Commons Attribution International 4.0 License. SBES '20, October 21-23, 2020, Natal, Brazil

(C) 2020 Copyright held by the owner/author(s).

ACM ISBN 978-1-4503-8753-8/20/09.

https://doi.org/10.1145/3422392.3422482
In 34th Brazilian Symposium on Software Engineering (SBES '20), October 21-23, 2020, Natal, Brazil. ACM, New York, NY, USA, 10 pages. https: //doi.org/10.1145/3422392.3422482

\section{INTRODUCTION}

Software Engineering (SE) is a topic that is faced with both apprehension and excitement by undergraduate students all over the world. The amazing possibilities opened by the transition from individual programming into development of software as an engineering activity performed inside a development team raise a myriad of questions both from students and instructors. The latter strive to find the right balance between the more theoretical concepts such as software life cycle models to the more practical such as configuration management tools such as git ${ }^{1}$, which are basic competences of the modern software engineer. In the midst of this, teamwork issues cannot be ignored, as modern software development is at its root a team activity. Delivering this diversity of competences in breadth and depth in the context of a graduation course continues to be a challenge for educators, and the adoption of project-based learning has been recognized a very valuable approach [1].

Project-based learning (PBL) allows for the close intertwining of theory and practice in the context of a project that needs to be developed as a team activity, according to the concepts and competences being acquired by students, while dealing with all the organizational and people issues involved.

However, PBL itself raises new issues that need to be addressed to achieve its full potential: the project needs to be significant to be able to exercise as much as possible the topics in the syllabus, its complexity cannot be overwhelming to the still inexperienced students, and foremost, each individual should receive the attention most adequate for its specific needs.

Addressing all these concerns raises the problem of scalability: project-based learning is very demanding to the instructors due to its inherent diversity of unique situations, multiplicity of topics and technologies addressed, and always novel challenges whenever a new project with a new course starts. It is a challenge to adopt PBL in a class of 15 to 20 students, and we have applied it to a software engineering course with more than 200 students for more than six years. In our context the scalability issues are exacerbated by the diversity of students' backgrounds since they are enrolled in different majors.

In this paper, we describe how we have successfully addressed the scalability issues of PBL in the context of a large and heterogeneous $\mathrm{SE}$ course. A central and distinctive aspect of our approach is that we have leveraged a state-of-the-art low-code development platform,

\footnotetext{
${ }^{1}$ https://git-scm.com/
} 
in collaboration with an industrial partner which was involved not only as technology provider as well as the client for the project.

According to the specificity of our context, the goals we have set for our course are as follows:

(1) to level an heterogeneous group of students, coming from different degrees and majors, regardless of prior education or experience in a given technology or programming language; that is to say that the project should be designed not to favor students with greater technical experience or training, while still being possible to implement in one semester;

(2) to reduce the gap between the individual performance of a student and the global assessment/grade of a potentially large group of students; this goal should be achieved by providing adequate individual feedback while still providing students a large-group working experience, i.e., it should not be achieved with the overhead (unbearable for the faculty) of having students working in small groups;

(3) to cope with a large number of students having the experience on how to develop a real software product for an actual industrial client; having an industry client also means that the requirements for the project are not (completely) under control by the faculty, which raises additional complexity and concerns for the project implementation.

With this set of main goals in mind, ultimately we seek to provide an answer to the following research question:

$\mathcal{R Q}$ : Is it possible to leverage a low-code approach to support a Software Engineering Course using Project-Based Learning, that is pedagogically and successfully implemented in an heterogeneous and large scale context?

We have assessed the merit of this approach using two complementary perspectives.

For once, we used the grades obtained by students throughout the project as a proxy of their progress and learning achievements. The grades received input from both the instructors and, even if in a smaller extent, the client. Our analysis targets both the individual and the group performances.

Secondly, we leverage the pedagogical surveys that are implemented by our University as a way to collect feedback from students regarding the courses they take. In the particular case of our course, this provided a significant perspective, representing over $70 \%$ of the enrolled students.

The complementary analysis of these approaches provides strong evidence that: i) students were able to achieve a performance in line with the expectation of the instructors and the client; and, ii) the course, in which the project plays a central role, was well acknowledged by the students engaging in it.

Notably, we have witnessed that students majoring in different topics performed comparatively well. This consists of a significant difference when compared to previous editions of the same course.

This paper is organized as follows. In section 2 the low-code platform adopted to provide scalability while providing a personalized education is presented. In section 3 we present a detailed description of the academic context concerning the software engineering course object of this study, including some details of the teaching and grading methodologies applied. In section 4 we provide an overview of the project adopted in the last academic year $(2019 / 20)$ as an example of the type of real-world projects that our undergraduate SE students are expected to complete in one semester. A discussion of the observations and results achieved are presented in section 5 . The threats to validity of these results are presented in section 6 . We then present, in section 7, a survey of related work and how it compares to our approach. Finally, section 8 concludes the paper by summarizing the major observations and the improvements intended for this course in the next academic year.

\section{THE LOW CODE PLATFORM}

A central and distinctive characteristic of our project-based approach to SE education was the adoption of a low-code approach in order to achieve scalability. In this section, we describe the OutSystems platform ${ }^{2}$ for low-code development that was adopted in this SE course, as the tool that allowed us to scale the course, provide a team based learning context, while providing quality, personalized student guidance.

These days, many low-code platforms have achieved a significant market presence, having been originated in many countries and created by many different companies. An evaluation conducted by Forrester [9] identified the 13 most significant suppliers, among which feature Appian, Kony, Mendix, Microsoft, Salesforce and OutSystems.

While platforms provided by different companies have distinctive features and properties, there are benefits that are generally attributed to low-code as a development paradigm [5]:

- it provides integrated and easily customizable security, crossplatform support and data integration capabilities;

- it does not require previous knowledge and (vast) experience on specific programming languages or technologies;

- it allows the construction of multiple-platform applications simultaneously;

- it allows to develop, update and deliver new features in short time periods.

Focusing on OutSystems, the company was founded in 2001, and since 2016 it has remained the market leader in Low-Code Platforms for Application Development. ${ }^{3}$ The vision of the company is to facilitate the development of various types of software, allowing developers to create web and mobile applications using a high-level visual language.

The visual language provided by OutSystems allows developers to work on an abstraction level that allows to model and design different layers of the applications such as Data, Process and User Interface. An example of a Process model specified in OutSystems' modeling language is presented in Figure 1.

Once specified using visual models, applications are compiled and published in one click, which generates all the application code and all database assets, and allows their deployment into execution environments across multiple devices and over different stack combinations.

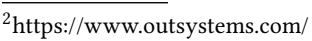

${ }^{3}$ https://www.outsystems.com/evaluation-guide/what-do-gartner-and-forrestersay-about-outsystems/
} 


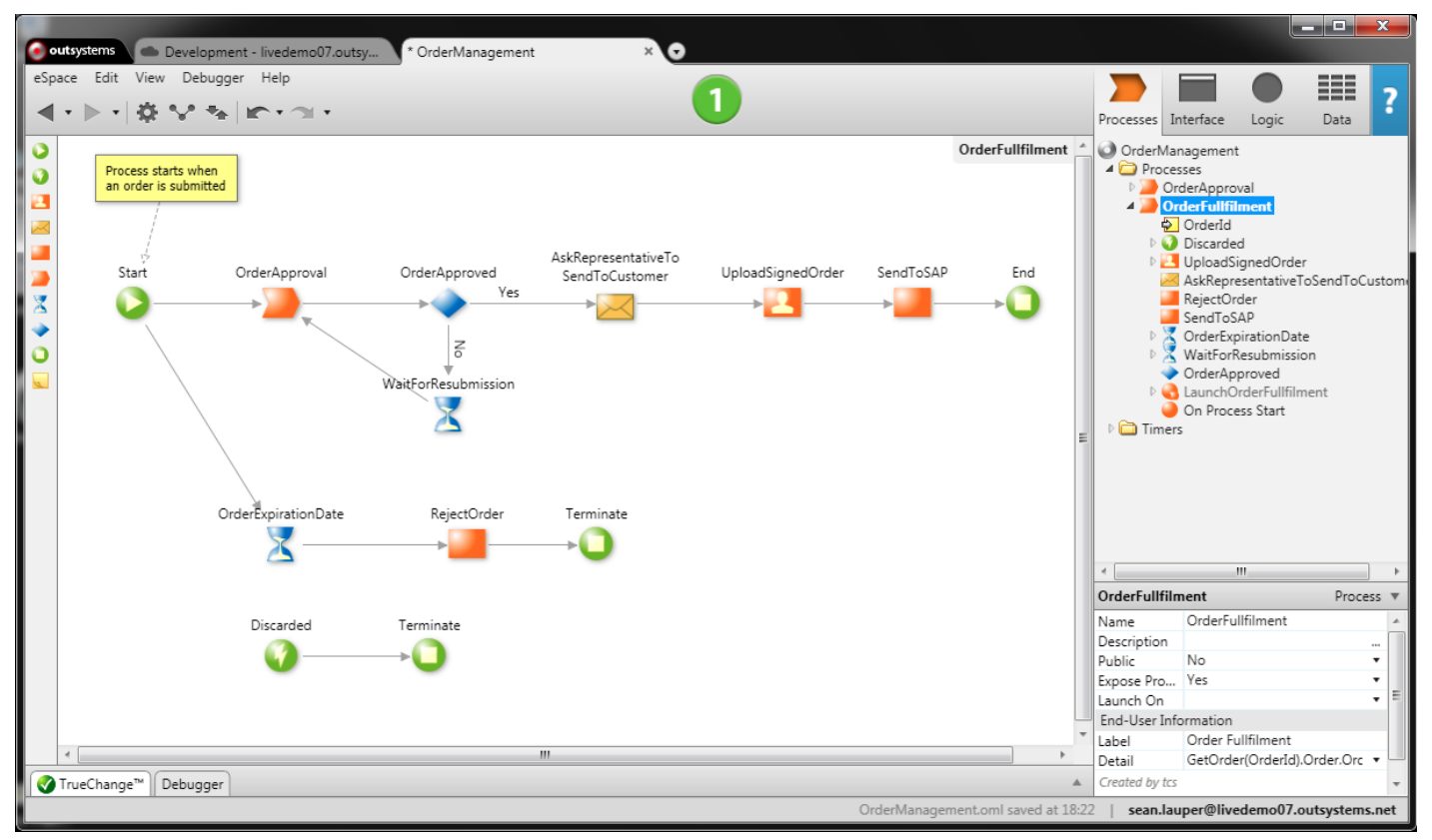

Figure 1: Example of Process Model written in OutSystems

Going back to the goals elicited in Section 1, we now describe how we believe the low-code platform allowed to implement the project characteristics we have defined in order to achieve them:

(1) to level an heterogeneous group of students, coming from different degrees and majors, regardless of prior education or experience in a given technology or programming language; By not assuming prior experience in a given programming language or a particular technology, the adoption of a low code platform played a decisive role here.

(2) to reduce the gap between the individual performance of a student and the global assessment/grade of a potentially large group of students;

As we will describe in more detail later, during project execution teams were split into sub-teams, which we refer to as forks. The introduction of team forks was (admittedly and publicly) announced as a motivational driver within the teams, so that members with similar goals and effort availability would end up grouping together. Managing the complexity associated with cloning the project and managing user access to the cloned repository in a centralized manner, and in a timely manner, was facilitated by the use of the functionalities provided by the platform to its clients. Indeed, as exemplified in Figure 2, the platform provides an integrated setting to manage, e.g., applications, users/developers and execution environments, and to monitor all the associated activity (e.g., all the applications being published by all students and their delta compared to the previous versions).

(3) to cope with a large number of students having the experience on how to develop a real software product for an actual industrial client;
Besides the management possibilities for instructors already described, the platform allowed students to achieve: i) highproductivity by using visual development to deploy and manage applications; ii) the ability to frequently deploy applications and update them with one click in a cloud environment; and iii) minimized effort associated with handling database scripts and deployment processes.

Ultimately, using the platform provided a controlled and integrated environment that facilitated the instructors' supervision and guidance of the work while also allowing students to focus more on the software engineering processes and less on (low-level) technical or technological aspects. We believe this is a desirable characteristic of a SE project, and one that we have actually sought also in previous editions of the course (without resorting to lowcode development). In this edition, the project particularly has an even stronger modeling component, as by the use of OutSystem's platform programs are generated automatically from abstract visual models that teams built throughout the entire development phase.

\section{COURSE DESCRIPTION}

In this section, we describe the Software Engineering course that we were responsible for and that is the object of the observations provided in this paper. This Software Engineering course is being provided for undergraduate students at Universidade de Coimbra for more than 10 years, having adopted project-based learning (PBL) about seven years ago under the supervision of one of the authors. Since its early adoption it was manifest that PBL was the right methodology for this course due to its intrinsic intertwining of theory and practice in the context of a real software project, which motivates the students while exposing them to the challenges of having a real client. 

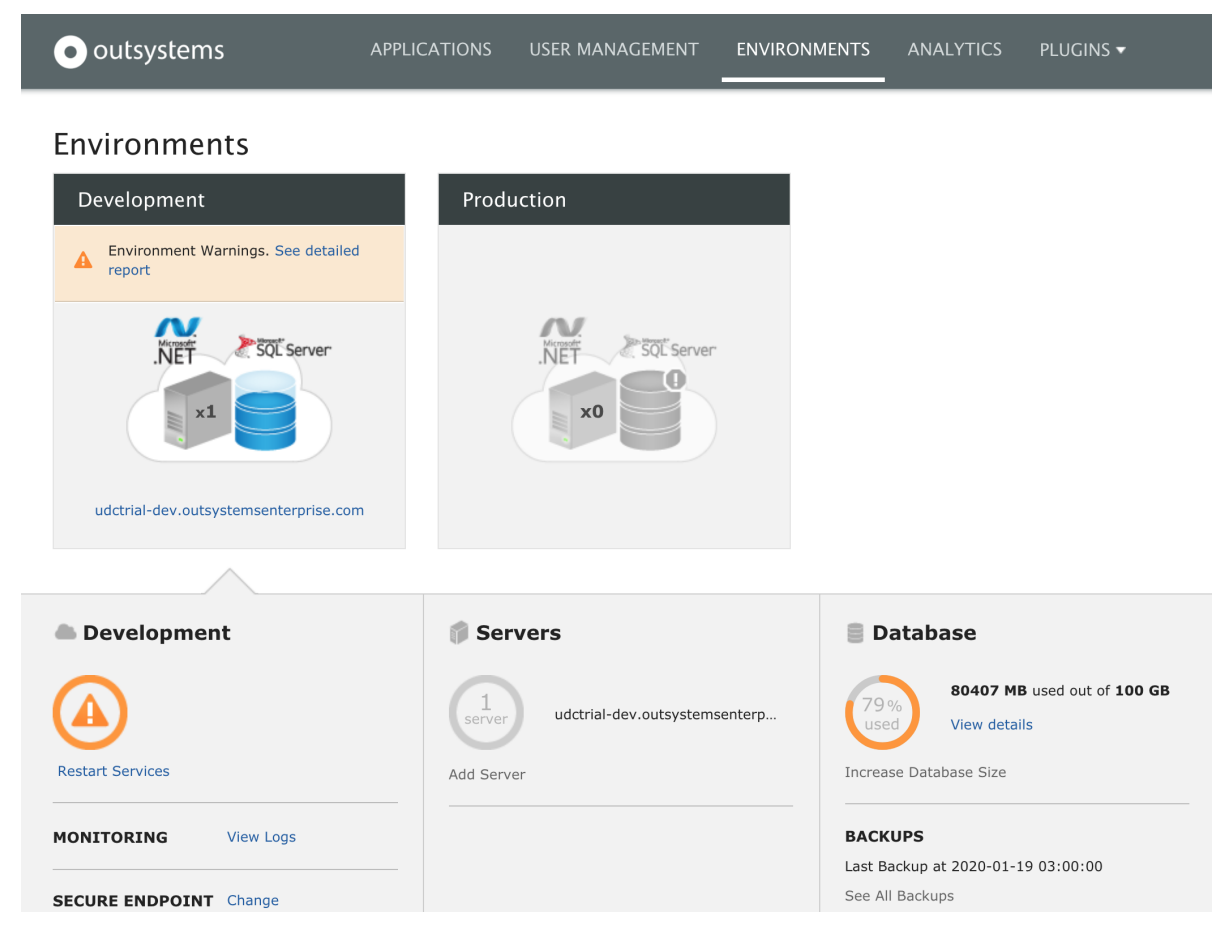

Figure 2: Integrated Development Monitoring with Lifetime.

The course is offered in the $3^{r d}$ year, i.e., the graduation year, for the bachelor degrees where it is mandatory (namely BSc in Informatics Engineering and BSc in Design and Multimedia), or as an elective in the $1^{\text {st }}$ year for other master programs, for students from different backgrounds (e.g. MSc Electrical Engineering). The course assumes an individual effort for the course corresponding to $6 \mathrm{ECTS}^{4}$ credits i.e. a typical one semester course. The semester has a duration of fourteen weeks during which students take other courses in parallel, typically 4 if they are strictly following their degrees' curricula (and not, e.g., re-taking previous courses). Regarding the SE course, each student should attend a lecture for two hours and a laboratory session for another two hours and is expected to self-study up to four hours per week. Class attendance is not mandatory, but strongly advised. All the students are enrolled in the same lecture class.

The software project spans the entire semester and it accounts for $50 \%$ of total student academic load, acting as a laboratory where students apply knowledge gained from the lectures in a semi-realistic, mentored, environment. The remaining $50 \%$ grade is obtained on a written exam after the end of classes and after the project is delivered.

The projects are proposed by external partners, mostly private companies. A set of external industry partners contacted by the instructors submit a project proposal, and the most adequate to SE education purposes is selected by the course coordinator. We try to select projects that are a good fit to the whole course syllabus (from requirements to delivery), interesting for the students and relevant for the proposing local partner while not being critical

\footnotetext{
${ }^{4}$ European Credit Transfer and Accumulation System
}

to their business since this is an academic work. We receive and deploy a very diverse set of projects, from a Kanban dashboard for an IT helpdesk service (still in production today), to an Android app for a real estate company (the core students were hired to continue working on it), and even an information system extension to manage attendance in a school in Brazil (i.e. the client was in another continent).

The project is the same for all teams, so we have as many versions of the same project as the number of teams running in parallel. Each class of about 30 students starts as a single team (more about that later). It would be an understatement to say that the first 2 to 3 weeks are prone to some chaos inside the teams. This is intentional, and has an educational purpose: the goal is to illustrate by experience that no work can be performed without organization, without roles and without tools that support communication and information sharing amongst the team members. Therefore this is actually a team building project phase, as for most of our students, this is their first experience working in a large team, overcoming different backgrounds and having direct contact with a client. Some order arrives with a (simple) deliverable by the end of week three, mostly driven by the deadline than by planning.

Instructors assigned to each laboratory class provide team and individual guidance. Each class starts with the team delivering a structured presentation about the work performed in the previous week in the different project areas (management, product, quality). Templates are used to standardize these presentations and they evolve during the semester to include more project relevant topics (e.g. in the beginning the focus is on requirements and team organization, later if shifts to design, metrics, delivery). The instructors 
try to act as an external advisor and students are motivated to take decisions by themselves, but justify the rationale for the decisions taken. There are no 'wrong answers' only ineffective ones, and students are motivated to take risks and assess the impact of such decisions. We recommend structuring the team in functional units, specially at the beginning of the semester, to provide some structure to such a large number of members and to promote accountability. Deliveries are due every three to four weeks thus work is organized in sprints/time boxing.

Despite the pedagogic advantages of working in such a large team due to the stringent need to organize the project repository, define a set of rules for artifact numbering, and setup dedicated communication channels (all typical software engineering issues), we are faced every year with a few less involved students, because the large size of the team allows them to step back and not contribute much to the project. Individual accountability is hard because the number of students enrolled in this course has grown from about 60 to more than 200 in recent years. Also their background became more diverse, as now we have students from different majors. This has raised a serious scalability problem, as the number of instructors did not follow the growth in number of students. Therefore a few years ago we adopted Gitlab ${ }^{\circledR}$ to be able to assess individual student contribution to the project. This brought additional education benefits, as such repositories are today mandatory in software development organization and students get their first hands-on experience with such platforms.

However, we came to realize that with the continuously increasing number of students even this was not enough. In fact the project repository interaction is not enough to assess student contributions as it lacks observability of a few relevant activities (e.g. management activities) and requires a 'policing' mindset from instructors that we try to avoid as much as possible. Therefore we complemented the git monitoring activity with regular project forks. At least twice in the semester, at a sprint transition, each team forks in two. Therefore by the end of the semester we have 4 teams per class, with about 6-7 students per team. The students decide among themselves who will be part of each team at the forking points. This strategy promotes active student involvement in the first part of the project (nobody wants to be in the 'leftover' teams) as well as in the final sprint because peer pressure is most effective in a small six-member team. The elegance of this solution is that the same platform that is being used for software project management (git), is also used to support the pedagogical goals. The adoption of these professional tools support the scalability of the course while also providing individual student activity monitoring. The simple fact that every contribution to the project is logged by these platforms act effectively as deterrent for academic fraud. At the same time it provides the instructors with the possibility of assessing the difficulties faced by individual students, thus promoting a more personalized guidance. In the last week of classes each team delivers a final public presentation describing the project, the product, and the software engineering issues involved before their peers, faculty members and the external clients. This final presentation is recorded and live streamed so that others (including family members) can watch it online. The intention is to provide relevance to the final presentation, so that students are committed to the project and focused on the goals.
In the last edition of this SE course (2019/20) we had 223 enrolled students, coming from 3 different degrees: 132 students from the Bachelor Degree in Informatics Engineering, 62 from the Bachelor Degree in Design and Multimedia and 29 students coming from the Master in Electrical and Computer Engineering.

There were 8 available laboratory options for students to choose from. After choosing one class, it is not possible for students to re-schedule or change it. We ended up with 4 laboratory classes each with 31 students enrolled, and 4 classes with 30, 20, 26 and 12 students enrolled, respectively.

At our University, enrolling in a laboratory class is done online, and once the signing up period opens, students rush to apply trying to enroll in the classes most convenient to their schedules. This introduces some randomness in the allocation of students per class, and all the classes but one ended up having students from the 3 degrees. ${ }^{5}$ As we discuss later, we leverage this property to assume that students were evenly distributed among classes, namely from a prior-education point of view.

\section{PROJECT DESCRIPTION}

In the context of this SE course, in the last academic year (2019/20) we have selected and implemented a project that is described in this section.

The project was structured for the entire duration of the course, as pictured in Figure 3. The first five weeks are used to address the team-building issues as referred previously and produce the first formal artifact, the Vision Document [4]. The development phase of the project, where the low-code platform is used, starts on the $6^{\text {th }}$ week.

The external client for the project was OutSystems, a global company that develops and commercializes a low-code development platform, which also served the project as technology provider.

The project was presented by OutSystems on the $2^{\text {nd }}$ week in a lecture class so to include all students at once. The presentation was, using different means, widely announced to students which attended it massively. The product that was commissioned by the client was a Recruitment Application that allows any company to hire easily and fast. The perils of the current recruitment processes and tool support were introduced, and the high-level requirements for the product were presented:

- Candidates must be able to apply to any open position;

- Artificial Intelligence algorithms should be used in order to rank the candidates that applied to a job application;

- Provide visibility to candidates about their current process statuses;

- Use social media to increase the visibility of the openings.

From this high-level description of the product to develop, the first challenge for students was then to refine it into a Vision Document. For this, weeks 3 to 5 were planned, after which students received concrete feedback and suggestions for improvement both from the instructors and the client. In this period, each laboratory class corresponded to one working group, so most students were working in (heterogeneous) groups of circa 30 students. As we have mentioned before, we believe that the process for students to sign

\footnotetext{
${ }^{5}$ The single exception did not have students from the Master in Electrical and Computer Engineering.
} 


\begin{tabular}{|c|c|c|c|c|c|c|c|}
\hline Week 1 & Week 2 & Weeks 3, 4 \& 5 & Weeks 6 \& & Weeks 8 \& 9 & Weeks 10 \& 11 & Weeks 12 \& 13 & Week 14 \\
\hline \hline $\begin{array}{c}\text { Course } \\
\text { Presentation }\end{array}$ & $\begin{array}{c}\text { Project } \\
\text { Presentation }\end{array}$ & $\begin{array}{c}\text { Vision } \\
\text { Document }\end{array}$ & Sprint 1 & Sprint 2 & Sprint 3 & Sprint 4 & Final \\
Pitch \\
\hline \hline
\end{tabular}

Figure 3: Project Planning

up for a particular class introduced an important randomness effect in the constitution of groups, which is a desirable effect to ensure fairness among working groups.

Having delivered the Vision Document, each group was asked to fork into two. This division was repeated half-way through the development phase. ${ }^{6}$ Both times, the following methodology was used: lecturers announced in advance a concrete moment after which they were available to receive, by email and per working group, the constitution of a (new) group composed of half of the members of the previous one (modulo one). This means that, e.g., an initial group of 28 students was forked into two groups of 14 elements and later each such group of 14 elements was forked intro two groups of 7 elements. The first email received per working group defined both groups for the following phase: the one with the listed elements, and the one with the remaining ones. Both groups forked from the same group inherited all the artifacts produced by the group thus far.

While in the construction of the Vision Documents groups were recommended to work under a simple time-boxed schedule, the development phase adopted an agile approach. Development was distributed in 4 two-week sprints, in which groups could decide which concrete development methodology to explore, and not only among the ones that were introduced in the lectures. Teams are allowed to self-organize, and during development most of them ended up using SCRUM-inspired methodologies, while some others adopted Kanban-inspired approaches. In any case, both the adopted methodology and the roles played by each student were discussed with the instructors. Throughout the different forks, teams were strongly advised to assign roles to each team member that were different from the ones they had already experienced.

The development phase included weekly review meetings among all the members of each team. These meetings were held, at least once per week, during the corresponding laboratory class, where the weekly progress was also described to and discussed with the instructors as referred above.

Sprint review meetings were held for sprints 1 to 3 and a pitch session was organized at the end of the development phase. The sprints and the pitch were taken as the primary input for the project assessment. Sprints 1 and 3 were assessed by the lecturers, and the half-life (after sprint 2) and final progresses were assessed at the same time by the lecturers and the client. The Vision Document accounted for $20 \%$ of the project grade, and the performance in the development phase, namely in sprints 1 to $4,15 \%, 20 \%, 15 \%$ and $30 \%$ of the grade, respectively.

Weekly and sprint review meetings consisted of decisive mechanisms to control and compare progress, both within each team as well as among different teams.

\footnotetext{
${ }^{6}$ Actually, given its reduced initial size, the smallest group forked in two only once, half-way through the development phase.
}

Besides being available in person in the half-life and final product presentations, the client was available online throughout the entire project. This was achieved through a Slack workspace that was created by the instructors and that the students and the client joined. In this workspace, teams created additional (private) channels to support communication among their members.

Finally, the project had a clear technical requirement: the products were to be developed using the OutSystems low-code platform.

We surveyed students and it was confirmed that: i) most had not heard about OutSystems prior to the course, and ii) none had prior experience with using the platform. This validated the reasonableness of our request of allocating effort in the project for all students to (equally) train themselves in the underlying technology. For this, the first 3 weeks of the project were assigned (the first two in full dedication and the third overlapping with the first vision documentrelated duties). Students were instructed to individually follow the guided learning path Becoming a Web developer ${ }^{7}$ provided by the OutSystems training resources, and namely its first two modules: the OutSystems Overview (estimated for a 40 minutes effort) and the Developing Web Apps (estimated for a 24 hours effort).

In the next section, we describe and analyze the results obtained by students in the project that we have thus far described.

\section{RESULTS AND ANALYSIS}

In this section, we describe the efforts we have conducted in order to assess the merits of our interpretation of PBL, by adopting a low-code platform, in the context of a SE course. In section 5.1, we analyze the performance of students throughout the project, under the lenses of the evaluation made by the lecturers and the client, and both within working groups and individually. In section 5.2, we leverage the course-assessment survey provided by our University after each course is delivered. The aim of this survey is to collect feedback from students and it is included in the overall qualityassessment methodology of the University.

\subsection{On the performance of students}

In this section, we analyze the performance of the students during the development phase of the project described earlier. Our analysis is based both on the grades that groups obtained in each sprint, as well as on the grades that students obtained individually.

In Figure 4, we present the grade each group obtained in all the 4 sprints of the development phase. ${ }^{8}$ The grades reflect the global group performance, while occasionally some students, in the vast majority of cases by mutual agreement, did not receive the grade obtained by the group in one particular sprint, e.g., due to absence of contribution. ${ }^{9}$ This confirmed in practice that students

\footnotetext{
${ }^{7}$ https://www.outsystems.com/learn/paths/2/becoming-a-web-developer/

${ }^{8}$ Please note that when development began, all groups but one had already been forked.

${ }^{9}$ In any case, the individual grade in a sprint was either the group grade or zero.
} 
co-assumed the responsibility of monitoring the group individual activity, as we anticipated and wished for.

\begin{tabular}{|c|c|c|c|c|c|}
\hline Group & Sprint 1 & Sprint 2 & Group & Sprint 3 & Sprint 4 \\
\hline \multirow{2}{*}{1.1} & \multirow{2}{*}{$27 \%$} & \multirow{2}{*}{$76 \%$} & 1.1 .1 & $66 \%$ & $61 \%$ \\
\hline & & & 1.1 .2 & $60 \%$ & $73 \%$ \\
\hline \multirow{2}{*}{1.2} & \multirow{2}{*}{$59 \%$} & \multirow{2}{*}{$78 \%$} & 1.2 .1 & $83 \%$ & $86 \%$ \\
\hline & & & 1.2 .2 & $73 \%$ & $60 \%$ \\
\hline \multirow{2}{*}{2.1} & \multirow{2}{*}{$84 \%$} & \multirow{2}{*}{$75 \%$} & 2.1.1 & $81 \%$ & $80 \%$ \\
\hline & & & 2.1 .2 & $77 \%$ & $73 \%$ \\
\hline \multirow{2}{*}{2.2} & \multirow{2}{*}{$89 \%$} & \multirow{2}{*}{$93 \%$} & 2.2 .1 & $96 \%$ & $96 \%$ \\
\hline & & & 2.2 .2 & $84 \%$ & $81 \%$ \\
\hline \multirow{2}{*}{3.1} & \multirow{2}{*}{$79 \%$} & \multirow{2}{*}{$72 \%$} & 3.1 .1 & $54 \%$ & $75 \%$ \\
\hline & & & 3.1 .2 & $47 \%$ & $0 \%$ \\
\hline \multirow{2}{*}{3.2} & \multirow{2}{*}{$87 \%$} & \multirow{2}{*}{$71 \%$} & 3.2 .1 & $86 \%$ & $82 \%$ \\
\hline & & & 3.2 .2 & $80 \%$ & $77 \%$ \\
\hline \multirow{2}{*}{4.1} & \multirow{2}{*}{$79 \%$} & \multirow{2}{*}{$78 \%$} & 4.1 .1 & $64 \%$ & $79 \%$ \\
\hline & & & 4.1.2 & $50 \%$ & $71 \%$ \\
\hline \multirow{2}{*}{4.2} & \multirow{2}{*}{$72 \%$} & \multirow{2}{*}{$58 \%$} & 4.2 .1 & $42 \%$ & $68 \%$ \\
\hline & & & 4.2 .2 & $44 \%$ & $57 \%$ \\
\hline \multirow{2}{*}{5.1} & \multirow{2}{*}{$66 \%$} & \multirow{2}{*}{$63 \%$} & 5.1 .1 & $66 \%$ & $83 \%$ \\
\hline & & & 5.1 .2 & $43 \%$ & $43 \%$ \\
\hline \multirow{2}{*}{5.2} & \multirow{2}{*}{$45 \%$} & \multirow{2}{*}{$62 \%$} & 5.2 .1 & $65 \%$ & $62 \%$ \\
\hline & & & 5.2 .2 & $64 \%$ & $62 \%$ \\
\hline \multirow{2}{*}{6.1} & $87 \%$ & $81 \%$ & 6.1 .1 & $94 \%$ & $96 \%$ \\
\hline & $07 \%$ & $01 \%$ & 6.1 .2 & $86 \%$ & $84 \%$ \\
\hline 62 & $34 \%$ & 8 & 6.2 .1 & $72 \%$ & $87 \%$ \\
\hline 6.2 & $34 \%$ & $85 \%$ & 6.2 .2 & $56 \%$ & $78 \%$ \\
\hline 71 & $71 \%$ & & 7.1 .1 & $65 \%$ & $87 \%$ \\
\hline 7.1 & $71 \%$ & $80 \%$ & 7.1.2 & $63 \%$ & $66 \%$ \\
\hline 70 & $62 \%$ & 600 & 7.2 .1 & $61 \%$ & $60 \%$ \\
\hline 1.2 & $62 \%$ & $68 \%$ & 7.2.2 & $61 \%$ & $65 \%$ \\
\hline 8 & 620 & 320 & 8.1 & $67 \%$ & $61 \%$ \\
\hline 0 & $02 \%$ & $33 \%$ & 8.2 & $51 \%$ & $0 \%$ \\
\hline
\end{tabular}

Figure 4: Development Phase Group Grades

We used a Shapiro-Wilk test to assess whether the grades obtained by groups in each sprint follow a normal distribution. We confirm this is not the case with a significance level of 0.05 , having obtained a p-value of $9.03 \mathrm{e}-6$. The grades had a median of $71 \%$ and an average of $68.11 \%$.

Complementary, we counted the occurrences of grades in intervals of $10 \%$. This count is plotted in Figure 5, which shows that the grades have a long left tail distribution, which means more students obtained good grades.

Combining the information from the statistical test and the plot, we realize that the group grades do not follow a normal distribution, and that there seems to exist a tendency in favor of the good grades. This is an indicator that groups had an overall satisfactory performance on the project.

We have used another Shapiro-Wilk test, this time to assess whether the individual grades obtained by students in the project follow a normal distribution. This was also not the case, having obtained a p-value of $1.66 \mathrm{e}-11$. The grades had a median of $69.80 \%$ and an average of $64.59 \%$.

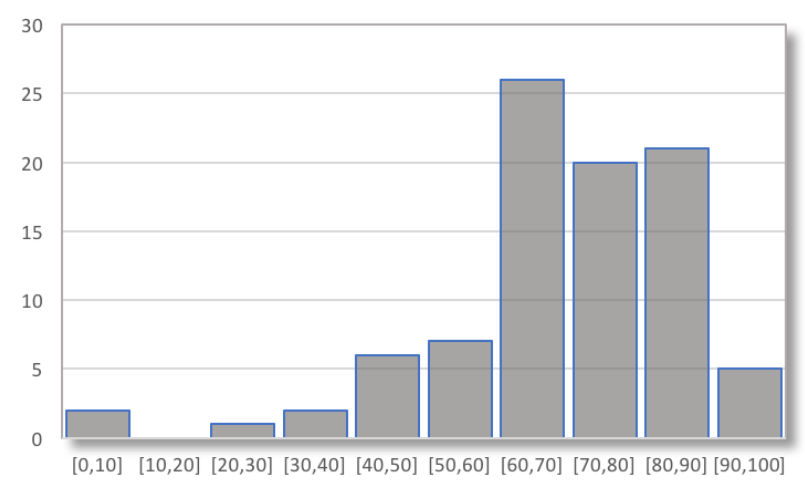

Figure 5: Distribution of Group Development Grades

Again, we have plotted the occurrence of individual grades in intervals of $10 \%$. This information is presented in Figure 6, and shows that individual grades also have a long left tail distribution.

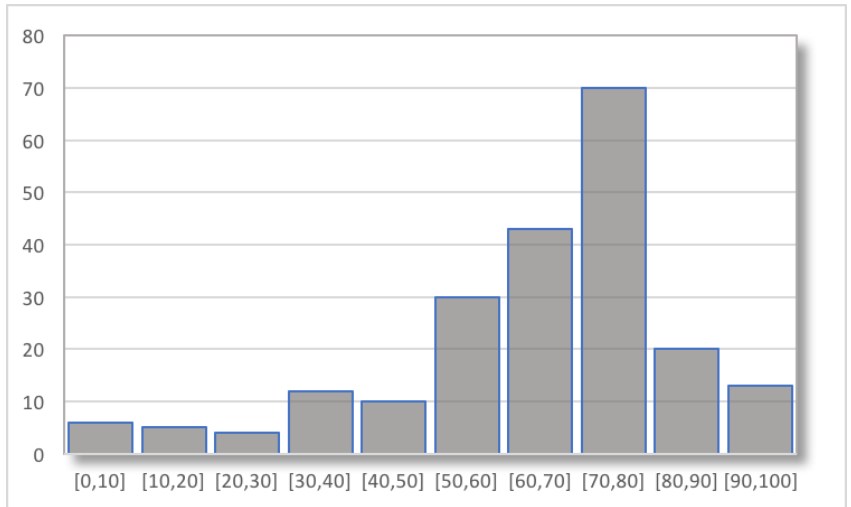

Figure 6: Distribution of Individual Development Grades

We believe that evidence exists, both: i) per group and sprint, and ii) individually and globally, that students were able to produce software in line with the needs of their client.

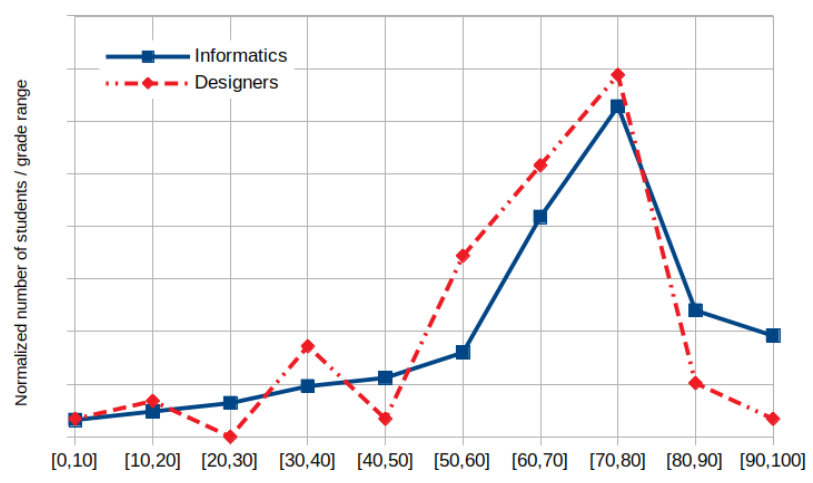

Figure 7: Normalized grades per background. 
But maybe the most revealing observation is depicted in Figure 7 , where the normalized grades (per number of students) for the two bachelor majors, Informatics Engineering (informatics) and Design and Multimedia (designers), show that the design students, which normally struggle with the ES course have actually performed remarkably well. This is a significant deviation from previous editions and a very strong hint that the adoption of the low-code platform, by not requiring a previous strong technical background, actually levelled the competences of these two very diverse audiences. The curve also shows that the highest grades keep being most frequent in the more technical students, as would be expected.

This observation addresses directly our first goal, concerning the adoption of a low-code platform to leverage a fair learning context for heterogeneous audiences.

\subsection{On the assessment of the course by students}

In order to monitor the quality of the teaching activities it provides, our institution has implemented a package of quality assessment methodologies. These methodologies include a survey that is offered to students at the end of each of the courses they undergo. ${ }^{10}$ In this section, we present and analyze the survey answers provided by the students enrolled in the Software Engineering course in which our project took place.

At our institution, answering surveys is anonymous and not mandatory, but students are strongly encouraged to leverage this possibility in a systematic way. The survey was provided before the examination period began, but after the project grades had already been made public.

It is important to clarify that, for standardization, surveys do not target specific theoretical or practical components of a course, bur rather the course from an overall perspective. This means that the institutional survey does not provide data that is specific to the project that we have designed and implemented, but instead provides data that includes a perspective on such project. While we considered providing a survey of our own, one that would be more specific to get feedback on the project itself, we refrained from doing so as this would potentially duplicate the answering effort, which is not feasible if generally adopted by all courses and might actually lead to less-committed answers being provided to the non-institutional surveys.

Although the SE course and its project were in practice exactly the same, the course was administratively processed as two different courses: i) one for the master students and ii) another for the bachelor students. The data we provide in this section therefore holds this same distinction.

As mentioned before, in the description presented in Section 3, the SE course had 223 enrolled students: i) 29 master students and ii) 194 bachelor students. The survey was answered by: i) 22 , so roughly $76 \%$ of the master students and ii) 139 , so roughly $71 \%$ of the bachelor students. These rates are significant to provide further insights on the course and its execution.

\footnotetext{
${ }^{10}$ To the best of our knowledge, this is a common practice among higher-education institutions.
}

The survey includes one question to assess whether: ${ }^{11}$

(1) The imposed effort is adequate.

The answers provided to this question are provided in Figure $8 .{ }^{12}$

\begin{tabular}{|c|c|c|c|c|}
\hline $\begin{array}{c}\text { Student } \\
\text { Group }\end{array}$ & Light & Adequate & $\begin{array}{c}\text { Moderately } \\
\text { Heavy }\end{array}$ & Excessive \\
\hline \hline Master & $0 \%$ & $63 \%$ & $32 \%$ & $5 \%$ \\
Bachelor & $1 \%$ & $38 \%$ & $32 \%$ & $26 \%$ \\
\hline \hline
\end{tabular}

\section{Figure 8: Percentage of Ratings per Effort Category}

We can see that most master students acknowledge an adequate effort associated with the course, while circa $1 / 3$ acknowledge a moderately-heavy effort. In the case of bachelor students, we can also observe that adequate and moderately-heavy were the most selected categories, but circa 1/4 of the students report an excessive effort, which is relevant.

Analyzing these results, we may draft a first insight. While bachelor students globally referred an appropriate effort for the project, master students were either potentially more productive or assumed that a higher effort was required to successfully complete the project.

Furthermore, the survey includes the following statements:

(2) Globally, the learning outcomes were of quality.

(3) The perceived learning outcomes that students were able to achieve were satisfactory.

(4) The perceived student engagement in the learning processes was satisfactory.

(5) The perceived development of analysis and critical reflection competences by the students was satisfactory.

(6) Globally, my performance during the course was satisfactory.

These statements were then confirmed/disproved by students in a Likert scale, where 1 provides a Totally Disagree statement and 5 provides a Totally Agree statement. Actually, the survey includes other statements that we are not including here as their analysis does not seem to be useful in the context of this paper. ${ }^{13}$

The responses that were provided by students to Statements (2)-(6) were unified as weighted averages, whose results we present in Figure 9.

The presented results show an overall good to very good appreciation of the course by the students, with the scores ranging from 3.5 to 4.4 in a scale up to 5 . Still, they seem to suggest a higher global appreciation by master students when compared to bachelor students. This may potentially reflect a more mature vision by the master students or a more extensive education/training record.

In any case, we believe the students' feedback confirms that our approach was successful, even in the challenging context that we have described earlier.

In the next section, we describe validity threats that may affect our analysis and findings described in this paper.

\footnotetext{
${ }^{11}$ The survey is provided in native language other than English, and despite our best effort, our translation may not entirely reflect our University's intention for the text of the survey.

${ }^{12}$ Please note that students may opt not to provide an answer to a question, hence totals may not be of $100 \%$.

${ }^{13}$ One example of a statement we are not including is The bibliographic references that were provided were adequate.
} 


\begin{tabular}{|c|c|c|}
\hline Question & Student Group & Weighted Average \\
\hline \hline Globally, the learning outcomes were of quality. & Master & 4.0 \\
& Bachelor & 3.5 \\
\hline The perceived learning outcomes that students were able to achieve were satisfactory. & Master & 4.0 \\
Bachelor & 3.6 \\
\hline The perceived student engagement in the learning processes was satisfactory. & Master & 4.4 \\
& Bachelor & 4.0 \\
\hline The perceived development of analysis and critical & Master & 4.2 \\
reflection competences by the students was satisfactory. & Bachelor & 3.9 \\
\hline Globally, my performance during the course was satisfactory. & Master & 4.2 \\
& Bachelor & 3.8 \\
\hline \hline
\end{tabular}

Figure 9: Weighted Average of Answers per Survey Question, on a 1-5 Scale

\section{THREATS TO VALIDITY}

We have used the performance of the students, and namely the grade they obtained in the project, to assess the educational merit of the project model. While we are not exactly measuring what software engineering skills students really acquired, we believe there is a strong connection between such skills and the successful delivery of products to their client. Ultimately, while it does not necessarily reflect an on-budget delivery (in terms of the available person*hours), we believe it assesses at least the goals of an ontime and on-quality delivery. In addition, OutSystems just recently opened the possibility for the interested students in signing for the Associate Web Developer certification exam. This will soon provide, from a technical point-of-view, a better perspective on the individual learning outcomes for students.

Complementary to using the performance of students as an indicator of merit, we have also analyzed the results of the pedagogical survey offered to students after the implementation of the project and the course. While the survey definitely provided a representative view of the students' perspective, it is more generally about the course instead of being focused on its practical assignment alone. This means that the results of the survey can not be taken with full precision to reflect the students' assessment of the project, but actually provide a perspective that includes the project assessment in the perspective of students. We believe this potential threat is mitigated by the fact that the project consists of, and was always explicitly referred to students as, an essential and central component of the course. It is therefore, highly unlikely that an unsatisfactory project could lead to the globally good (quantitative) feedback provided by the students regarding the course.

In the project we defined, the technology supplier, OutSystems, acted also as the client of the project. While this may suggest a potentially biased assessment of the products being delivered, the fact is that most project assessment duties were imposed on the instructors (on all 4 sprints), and the participation of the client (in the end of sprints 2 and 4) in the assessment was always discussed with the instructors, a consensus having been reached. Still, it would be interesting to explore in the future a low-code supported project having an industrial client other that the technology supplier.

In the next section we present a review of references on projectbased learning in software engineering, as well on adopting lowcode platforms in such context.

\section{RELATED WORK}

The adoption of project-based learning using an external real-world problem to support engineering education is not new. The late James Tomayko, the driving force behind the Master of Software Engineering (MSE) at Carnegie-Mellon University as early as 1987 created such a hands-on software engineering course [10]. This reference is full of sensible advice even today. Since one of the authors of this paper was a team mentor in that MSE program a few years ago, that experience was the motivation behind the PBL methodology of this SE course.

In line with this PBL approach [2], [3], [8], [7], and [11] describe their experience of software engineering education using a real project with real clients. [12] present an systematic literature review on the usage of Design Sprints as a PBL approach, i.e. that involves the full design to deployment process. This extensive work analyzes how the PBL units that produce authentic software are executed and in no case was the use of low-code platforms referred.

In [1] a detailed description of a course structured in a way remarkably similar to ours is presented. All these experiences use extensively commercial resources such as cloud platforms (Gitlab, Github, Slack, Jira, etc.) that are provided free or using academic licensing to provide students with real world tools while at the same time reducing the setup effort from instructors. To the best of our knowledge, however, we have not found any previous reference to the integration of low-code platforms in a real-world PBL context.

There have been several initiatives of using low-code tools in a educational context, e.g. [6], but these experiences have a very limited scope and do not involve neither professional low-code tools, neither real world projects. So while the above works either focus on low-code tools or in providing students with a realistic experience, both technically, working in a team, and dealing with a real client, we added to that context the usage of a professional low-code platform that somehow levels-up students with different backgrounds and programming proficiency. This approach promotes high-quality SE education, and supports scalability without compromising quality neither attention to the learning individual.

\section{CONCLUSIONS}

In this paper, we have described a project-based approach to a software engineering course, where the use of a low-code platform was a central feature. We were able to implement such an approach 
in a large and heterogeneous class, and in a project that had a real industrial partner.

We have assessed our initiative from two different angles: by inspecting the grades obtained by students, as an indicator of their learning progress, and by inspecting the feedback provided by students in a follow-up survey.

The performance of the students showed a positive tendency, whereas the survey complementary revealed that students generally assessed the course, including its project, as meritorious. The survey revealed a(n even) more positive perspective regarding our initiative from master students when compared to bachelor students.

So, going back to the research question we elicited in the introduction,

$\mathcal{R Q}$ : Is it possible to leverage a low-code approach to support a Software Engineering Course using Project-Based Learning, that is pedagogically and successfully implemented in an heterogeneous and large scale context?

we believe that our work and the content of this paper allow us to positively answer it. Actually, the grades were remarkably balanced amongst a large class of students with very different backgrounds, and with a significant success ratio.

In fact, having our analysis revealed promising results, it is our intention to maintain this approach in the future. In the next academic year we will continue improving this course using the feedback from students and the lessons learned from the previous edition:

- Organize a workshop, on low-code technology, in the beginning of the course to improve the learning curve of the platform and allow students to focus even for a longer period in the software engineering tasks of the project;

- Require that time logging, a loose requirement currently, becomes mandatory so that effort maps can be generated to promote a reflection on the meaningfulness of Unified Process phases.

- Proceed definitely into the flipped classroom model: as part of our effort to scale up the course and reduce the faculty effort, we have now most of the course content online (videos, presentation slides, animations, documentation, FAQs, and discussion forums), students will start delivering their standardized status report during the lecture class, followed by discussion of issues and application of theoretical concepts amongst all students, while the professor will act as a moderator and 'external consultant'. This will promote sharing of experiences and cross fertilization of ideas between teams, something that we perceive is not happening now: each team is too focused on its own problems and not learning from each others' experiences. In this model the laboratory classes will be used for team meetings (having coincident out-ofclass schedules for every team member can be a challenge), and the faculty member present can provide more value to the team by providing guidance on specific issues raised by each team that they might not feel comfortable discussing during the common lecture class.

We strive to achieve quality, individual education in a team setting, for a large class in a real environment. The adoption of professional tools, such as low-code platforms on top of shared repositories seems to be an effective way to achieve such endeavour.

\section{REFERENCES}

[1] Bernd Bruegge, Stephan Krusche, and Lukas Alperowitz. 2015. Software engineering project courses with industrial clients. ACM Trans. Comput. Educ. 15, 4, Article 17 (Dec. 2015), 31 pages. https://doi.org/10.1145/2732155

[2] S. C. dos Santos, M. da Conceição Moraes Batista, A. P. C. Cavalcanti, J. O. Albuquerque, and S. R. L. Meira. 2009. Applying PBL in Software Engineering Education. In 2009 22nd Conference on Software Engineering Education and Training. 182-189.

[3] Maria Lydia Fioravantia, Bruno Sena, Leo Natan Paschoal, Laíza R Silva, Ana Paula Allian, Elisa Yumi Nakagaw, Simone R.S. Souza, Seiji Isotani, and Ellen Francine Barbosa. 2018. Integrating Project Based Learning and Project Management for Software Engineering Teaching: An Experience Report. In Proceedings of the 49th ACM Technical Symposium on Computer Science Education. ACM, New York, NY, pp. $112-117$.

[4] IBM. [n.d.]. Vision Document. https://www.ibm.com/support/knowledgecenter/ SSWMEQ_4.0.6/com.ibm.rational.rrm.help.doc/topics/r_vision_doc.html. Accessed: January 14, 2020.

[5] Jon Idle. 2016. Low-Code rapid application development - So, what's it all about? Platinum Business Magazine (2016), 52-53.

[6] Julie Kukesh and Kat Brandenburg. 2018. Innovative low-code tool for Systems Analysis and Design. (2018). http://aisel.aisnet.org/mwais2018/55.

[7] Dennis Pagano and Bernd Bruegge. 2013. User involvement in software evolution practice: A case study. In Proceedings of the 35th International Conference on Software Engineering (ICSE '13). IEEE, 953--962.

[8] Carolyn Rosiene and Joel Rosiene. 2006. Experiences with a real software engineering client. (2006), 12-14.

[9] John R. Rymer and Rob Koplowitz. 2019. The Forrester WaveTM: Low-Code Development Platforms For AD\&D Professionals, Q1 2019. Forrester (2019).

[10] James Tomayko. 1987. Teaching a Project-Intensive Introduction to Software Engineering. (1987). Retrieved from the Software Engineering Institute, Carnegie Mellon University website: http://resources.sei.cmu.edu/library/assetview.cfm?AssetID=10331.

[11] Vera King Vincent Cicirello and Farris Drive. 2013. Experiences with a real projects for real clients course on software engineering at a liberal arts institution. Journal of Computing Sciences in Colleges 6 (2013), 50-56.

[12] Edna Dias Canedo Vinícius Gomes Ferreira. 2020. Design sprint in classroom: exploring new active learning tools for project-based learning approach. fournal of Ambient Intelligence and Humanized Computing. 11, 3 (2020), 1191-1211. https: //doi.org/10.1007/s12652-019-01285-3 